\title{
Suficiência amostral para cravina de jardim cultivada em diferentes substratos ${ }^{(1)}$
}

\author{
SIDINEI JOSÉ LOPES ${ }^{(3)}$, MARÍLIA MILANI(2)*, \\ ALESSANDRO DAL'COL LÚCIO(3) e LINDOLFO STORCK ${ }^{(3)}$
}

\begin{abstract}
RESUMO
A cravina é uma planta excelente para compor jardins por possuir florescimento precoce, floração abundante e ótimo desempenho na primavera e outono. O objetivo do trabalho foi estimar o tamanho de amostra para plantas de cravina de jardim, cultivadas em diferentes substratos, e verificar a variabilidade do tamanho de amostra entre variáveis de crescimento e produção e substratos. Utilizaram-se sete tratamentos (substratos): $\mathrm{S} 1=50 \%$ solo $+50 \%$ cinzas de casca de arroz; $\mathrm{S} 2=80 \%$ solo $+20 \%$ húmus de minhoca; $\mathrm{S} 3=80 \%$ cinzas de casca de arroz $+20 \%$ húmus de minhoca; $\mathrm{S} 4=40 \%$ solo $+40 \%$ cinzas de casca de arroz $+20 \%$ húmus de minhoca; S5 $=100 \%$ turfa preta; S6 $=100 \%$ substrato comercial Mecplant ${ }^{\circledR} ; \mathrm{S} 7=50 \%$ turfa preta $+50 \%$ cinzas de casca de arroz, com 56 repetições cada um, totalizando 392 plantas de cravina de jardim, nas quais se avaliaram 17 variáveis de crescimento e produção. Utilizou-se a metodologia de reamostragem bootstrap, com reposição, para cada variável dentro de cada substrato, com erro preestabelecido de: 5, 10, 20 e 40\% da média (D\%). Para um intervalo de confiança de $95 \%$, com $\mathrm{D} \%=20$, o substrato com $50 \%$ de solo e $50 \%$ de cinzas de casca de arroz apresentou o maior tamanho de amostra em 11 variáveis; quando se comparou as variáveis, o número de botões florais apresentou o maior tamanho de amostra, em média 113 plantas. A utilização de amostras de 44 plantas de cravina de jardim para o substrato comercial Mecplant ${ }^{\mathbb{R}}$ atenderá a precisões inferiores ou iguais a $20 \%$, para todas as variáveis avaliadas. Há variação no tamanho de amostra em relação ao substrato utilizado e a variável avaliada em plantas de cravina de jardim.
\end{abstract}

Palavras-chave: Dianthus chinensis L., floricultura, planejamento experimental, amostragem.

\begin{abstract}
Sample sufficiency of chinese pink grown in different substrates

The cravina is an excellent plant to build up gardens due to its early flowering, abundant flowering and great performance in spring and autumn. The objective was to estimate the sample size for plant chinese pink, grown on different substrates, and check the variability of the sample size between growth parameters and production and substrates. They used seven treatments (substrates): $\mathrm{S} 1=50 \%$ soil $+50 \%$ rice husk ash; S2 $=80 \%$ soil $+20 \%$ earthworm castings; $\mathrm{S} 3=80 \%$ rice husk ash $+20 \%$ earthworm castings; S4 $=40 \%$ soil $+40 \%$ rice husk ash $+20 \%$ earthworm castings; S5 $=100 \%$ peat; S6 $=100 \%$

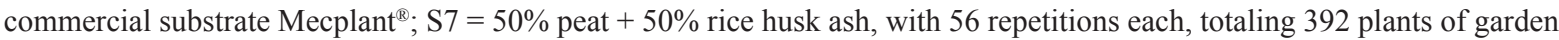
pink, which was evaluated in 17 of growth and production parameters. The methodology used to bootstrap resampling, with replacement, for each character within each substrate with predetermined error: 5, 10, 20 and 40\% of the average (D\%). To a $95 \%$ confidence interval, with $\mathrm{D}=20 \%$, the substrate $50 \%$ soil and $50 \%$ of rice husk ash had the largest sample size 11 characters; when comparing the characters, the number of flower buds had the highest sample size on average 113 plants. Using samples of 44 plant chinese pink for commercial substrate Mecplant ${ }^{\mathbb{R}}$ meet the lower precisions or equal to $20 \%$ for all variables. There is variation in sample size in relation to the substrate used and the variable evaluated in chinese pink plants.
\end{abstract}

Keywords: Dianthus chinensis L., Floriculture, Experimental planning, Sampling.

\section{INTRODUÇÃO}

A cravina (Dianthus chinensis L.) é uma planta utilizada para a composição em jardins, pois possui florescimento precoce e floração abundante e apresenta ótimo desempenho na primavera e outono (SAKATA SEED SUDAMERICA LTDA $\left.^{\circledR}, 2015\right)$.

A cravina de jardim é produzida em substratos até o ponto de comercialização (abertura do primeiro botão floral), quando então são destinadas aos centros comerciais para posterior uso nos jardins. No processo produtivo de mudas floríferas, um fator importante de estudo são as formulações de substratos, que devem apresentar baixo custo de produção e características físicas e químicas que permitam o pleno desenvolvimento da espécie (KÄMPF, 2005). As características principais de um substrato ideal para o cultivo de plantas floríferas e ornamentais são: disponibilidade em grande quantidade no mercado local, preço baixo e facilidade de manuseio (FARIA et al., 2001).

Em experimentos agrícolas, a mensuração em todas as plantas da área útil da unidade experimental é a técnica adequada para estimar a média do caráter em

\footnotetext{
(1) Recebido em 24/06/2015 e aceito em 03/02/2016

(2) Universidade Federal de Santa Maria (UFSM), Departamento de Fitotecnia, Centro de Ciências Rurais, Santa Maria-RS, Brasil. *Autor correspondente: mariliakmilani@gmail.com.

(3) Universidade Federal do Rio Grande do Sul (UFRGS), Departamento de Horticultura e Silvicultura, Porto Alegre-RS, Brasil.
} 
avaliação. Muitas vezes, no entanto, ocorrem limitações para avaliação de um grande número de plantas, como a disponibilidade de tempo, de mão-de-obra e de recursos financeiros. Nesses casos, a amostragem de plantas dentro da unidade experimental é uma alternativa adequada (CARGNELUTTI FILHO et al., 2010a).

Para obtenção de estimativas com a mesma precisão, há necessidade de estimar um tamanho de amostra específico para cada variável, genótipo e experimento (CARGNELUTTI FILHO et al., 2009). Diante disso é importante estimar um tamanho de amostra para cada situação de cultivo, evitando trabalhar com amostras super ou subdimensionadas.

O tamanho de amostra tem sido determinado por diferentes metodologias, porém as técnicas de reamostragem com reposição têm sido mais recentemente utilizadas para este fim (NUNES et al., 2006).

O tamanho de amostra já foi determinado para grandes culturas: soja (CARGNELUTTI FILHO et al., 2009), milho (STORCK et al., 2007; CATAPATTI et al., 2008), feijão (CARGNELUTTI FILHO et al., 2008); para frutíferas: laranjeira (BELASQUE JUNIOR et al., 2008) e goiabeira (ROZANE et al., 2009); e para olerícolas: abóbora italiana (SOUZA et al., 2002), pimentão (LORENTZ et al., 2005), cenoura (SILVA et al., 2009); e alface (SANTOS et al., 2007). No entanto, não há relatos na literatura da determinação do tamanho de amostra envolvendo as plantas ornamentais e suas variáveis de crescimento e produção, por isso a determinação de técnicas experimentais adequadas ao cultivo de cravina de jardim, tal como, o tamanho de amostra ideal, é importante para qualificar os resultados da pesquisa e para a redução do erro experimental.

Sendo assim, o objetivo do trabalho foi estimar o tamanho de amostra para plantas de cravina de jardim, cultivadas em diferentes substratos, e verificar a variabilidade do tamanho de amostra entre variáveis de crescimento e produção e os substratos.

\section{MATERIAL E MÉTODOS}

O experimento foi conduzido no período de 29 de abril a 29 de julho de 2011, em casa de vegetação, com cobertura de placas de policarbonato alveolar espessura de $10 \mathrm{~mm}$, e com $50 \%$ da interceptação da intensidade luminosa. A temperatura média do ar e a umidade relativa média do ar foram registradas diariamente, respectivamente, com termômetro de mínima de álcool, termômetro de máxima de mercúrio, e psicrômetro de material plástico e corresponderam a $18,6^{\circ} \mathrm{C}$ e a $68 \%$, respectivamente.

Utilizaram-se sete substratos escolhidos baseando-se na disponibilidade regional, ou na fácil obtenção e no baixo custo de aquisição. Alguns foram compostos pela mistura manual de componentes, e a proporção das mesmas foi feita pelo critério volume por volume. Os substratos (S) foram: $\mathrm{S} 1=50 \%$ solo $+50 \%$ cinzas de casca de arroz; $\mathrm{S} 2$ $=80 \%$ solo $+20 \%$ húmus de minhoca; $\mathrm{S} 3=80 \%$ cinzas de casca de arroz $+20 \%$ húmus de minhoca; $\mathrm{S} 4=40 \%$ solo $+40 \%$ cinzas de casca de arroz $+20 \%$ húmus de minhoca; $\mathrm{S} 5=100 \%$ turfa preta; S6 $=100 \%$ substrato comercial Mecplant ${ }^{\circledR} ; \mathrm{S} 7=50 \%$ turfa preta $+50 \%$ cinzas de casca de arroz

O solo foi obtido da coleta do horizonte B de um Argissolo vermelho, da Unidade de Mapeamento São Pedro, pertencente à região de Santa Maria. A cinza utilizada nas misturas dos substratos tem origem da queima da casca do arroz. O húmus foi obtido do minhocário do Departamento de Solos da UFSM, produzido por minhocas da espécie Eisenia andrei (BOUCHÉ, 1972), a partir de esterco curtido de bovinos criados em sistema de confinamento. Antes da instalação do experimento, o húmus foi peneirado em malha de $2 \mathrm{~mm}$. A turfa preta foi obtida da cidade de Araranguá, localizada no extremo sul do Estado de Santa Catarina (SC) e o substrato comercial Mecplant ${ }^{\circledR}$, constituído de casca de pinus, vermiculita, corretivo de acidez e fertilizantes, foi obtido no comércio local de Santa Maria, RS.

Utilizou-se o delineamento experimental inteiramente casualizado, com sete tratamentos (substratos), cada um composto de 56 plantas, escolhidas aleatoriamente através de sorteio, que caracterizaram as 56 repetições, totalizando 392 plantas avaliadas. As unidades experimentais foram sacos plásticos de polietileno na cor preta, com dimensões de $8,0 \mathrm{~cm}$ de altura, $8,0 \mathrm{~cm}$ de diâmetro, correspondendo a $402,12 \mathrm{~cm}^{3}$ de volume, com uma planta cada.

O material vegetal utilizado no experimento foi Dianthus chinensis L. 'Dianthus F1 Diamond', desenvolvido pela empresa Sakata $^{\circledR}$. As plântulas de cravina foram plantadas nos sacos plásticos com 28 dias após a emergência, aproximadamente, com três pares de folhas e $2,5 \mathrm{~cm}$ de altura. Foi realizada a irrigação manual, diária e localizada, mantendo a umidade do substrato próxima à capacidade de recipiente, até as avaliações finais do experimento (abertura do primeiro botão floral na haste principal). A água da irrigação apresentou valores médios de $\mathrm{pH}$ de $6,1 \mathrm{e}$ de condutividade elétrica de $110,08 \mu \mathrm{S} \mathrm{cm} \mathrm{cm}^{-1}$.

As propriedades físicas e químicas dos substratos foram avaliadas pelo laboratório de Análises de Rotinas da UFSM. As características físicas avaliadas foram: a densidade ( $\mathrm{g}$ $\left.\mathrm{cm}^{-3}\right)$, a porosidade total $\left(\mathrm{cm}^{3} \mathrm{~cm}^{-3}\right)$, o espaço de aeração $\left(\mathrm{cm}^{3} \mathrm{~cm}^{-3}\right)$ e a água disponível $\left(\mathrm{cm}^{3} \mathrm{~cm}^{-3}\right)$. As características químicas avaliadas foram: o potencial hidrogeniônico - $\mathrm{pH}$; o cálcio - Ca $\left(\mathrm{cmol}_{\mathrm{c}} \mathrm{dm}^{-3}\right)$; o magnésio - $\mathrm{Mg}\left(\mathrm{cmol} \mathrm{dm}^{-3}\right)$; a CTC efetiva $\left(\mathrm{cmol}^{\mathrm{c}} \mathrm{dm}^{-3}\right)$; a matéria orgânica - MO (m $\left.\mathrm{V}^{-1}\right)$; o fósforo - P-Mehlich $\left(\mathrm{mg} \mathrm{dm}^{-3}\right)$ e o potássio - K (mg $\mathrm{dm}^{-3}$ ) (Tabela 1).

Foram avaliadas 17 variáveis, por planta, para caracterizar o crescimento e desenvolvimento da cravina de jardim nos diferentes substratos. As variáveis avaliadas foram: 1) ciclo até a diferenciação: número de dias entre a repicagem e o início do estádio reprodutivo ou começo da formação do botão floral. 2) ciclo até o aparecimento do primeiro botão floral: período, em dias, da repicagem até o aparecimento do primeiro botão floral (com no mínimo tamanho igual a 0,5 cm). 3) ciclo de produção: período, em dias, da repicagem até o ponto de comercialização. Estas três primeiras variáveis apresentadas foram obtidas pela observação diária de todas as plantas desde o primeiro dia após a repicagem (DAR) até o término do experimento $90 \mathrm{DAR}$.

As demais variáveis foram determinadas aos 90 DAR, quando o primeiro botão floral da haste principal das mudas 
estava completamente aberto, sendo eles: 4) diâmetro da haste principal a $5 \mathrm{~cm}$ da base do substrato. 5) diâmetro da haste principal a $10 \mathrm{~cm}$ da base do substrato. $\mathrm{O}$ diâmetro da haste principal foi feito com auxílio de paquímetro digital, marca Digimess, e o resultado expresso em mm. 6) diâmetro da flor: correspondeu a medida do maior eixo horizontal das pétalas da primeira flor completamente aberta da haste principal, através de uma régua graduada em centímetros. 7) número de botões florais: contagem do número de botões florais da planta, com no mínimo tamanho igual a $0,5 \mathrm{~cm}$. 8) número de ramificações: contagem do número de ramificações da planta. 9) área foliar da haste principal. 10) área foliar das ramificações. 11) área foliar do total da parte aérea. A área foliar foi realizada através do método destrutivo, com equipamento modelo LI 3000C, da marca Licor, anotando o valor $\mathrm{em}^{2}$. 12) fitomassa verde da haste (folhas, flores e botões florais) principal. 13) fitomassa verde das ramificações. 14) fitomassa verde total da parte aérea. A fitomassa verde foi determinada através do uso de balança digital, com precisão de duas casas decimais (0,01 g). 15) fitomassa seca da haste principal. 16) fitomassa seca das ramificações. 17) fitomassa seca do total da parte aérea. A fitomassa seca foi feita a partir da secagem do material em estufa com ventilação forçada, à $40^{\circ} \mathrm{C}$, por sete dias (massa constante) (BARBOSA et al. 2010) e a determinação da massa foi feita em balança digital com precisão de mg.

Anterior ao cálculo do tamanho de amostra realizouse uma análise exploratória sobre o comportamento das distribuições de probabilidade das variáveis analisadas por substrato e por variável, pelo teste de Anderson-Darling e, ainda, da homogeneidade das variâncias dos substratos, pelos testes de Levene (quando os dados não atendiam ao pressuposto de normalidade) e Bartlett (dados com distribuição normal) em $5 \%$ de probabilidade de erro, utilizando o programa Action (ESTATCAMP, 2015).

O tamanho de amostra (número de plantas) foi determinado para cada substrato e variável avaliada, usando a metodologia de reamostragem com reposição, conforme aplicação de Storck et al. (2012).

Nos substratos com $50 \%$ solo $+50 \%$ cinzas de casca de arroz e com $80 \%$ cinzas de casca de arroz $+20 \%$ húmus de minhoca, os variáveis ciclo até a diferenciação e ciclo até o aparecimento do primeiro botão floral, foram avaliados com um número de plantas igual a 48 e 52, respectivamente, pois as demais se mantiveram no estágio vegetativo. Já para a variável diâmetro da flor, o número de plantas utilizadas no substrato com $50 \%$ solo $+50 \%$ cinzas de casca de arroz foi igual a 44 e, para a variável diâmetro da haste principal a 10 cm da base do substrato, o número de plantas foi igual a 16, pois as demais não apresentaram no momento da avaliação a flor aberta para mensurar o seu diâmetro e nem hastes com tamanho igual ou maior que $10 \mathrm{~cm}$.

A metodologia de reamostragem com reposição foi realizada para cada variável dentro de cada substrato. Foram geradas 3.000 reamostras por reamostragem, com reposição, para diferentes tamanhos de amostras $(\mathrm{k}=2$, $3,4, \ldots, 300)$. Portanto, para cada valor de $\mathrm{k}$, obteve-se 3.000 médias, que foram ordenadas para determinação dos valores dos percentis 2,5\% e 97,5\%. Após, calculou-se a amplitude do intervalo de confiança de $95 \%$, pela diferença entre o percentil $97,5 \%$ e o percentil $2,5 \%$. O tamanho de amostra foi determinado como sendo o valor de $\mathrm{k} \mathrm{em}$ que a amplitude do intervalo de confiança de 95\% (erro de estimação) foi igual a: 5, 10, 20 e 40\% da média (D\%). Para as simulações, foi utilizado um aplicativo elaborado em linguagem Pascal, obtendo as amplitudes e respectivos valores de $\mathrm{k}$.

\section{RESULTADOS E DISCUSSÃO}

Com relação às características físicas dos substratos (Tabela 1), pelos resultados de densidade do substrato, somente os substratos S3 e S7 apresentaram densidade suficiente para garantir estabilidade do recipiente $(0,27 \mathrm{e}$ $0,36 \mathrm{~g} \mathrm{~cm}^{-3}$, respectivamente). A faixa ideal para densidade dos substratos é entre 0,2 e $0,4 \mathrm{~g} \mathrm{~cm}^{-3}$, recomendada para recipientes de até $15 \mathrm{~cm}$ de altura. Valores acima desta faixa tornam o cultivo em recipiente mais difícil, principalmente pela limitação no crescimento das plantas, assim como, pela dificuldade no transporte dos vasos (KÄMPF, 2005). Portanto, os demais substratos apresentaram densidade fora da faixa ideal recomendada.

As necessidades quanto ao espaço de aeração variam conforme o tipo de planta, mas os valores referenciais na literatura situam-se entre 20 e 40\% (0,20 e 0,40 $\left.\mathrm{m}^{3} \mathrm{~m}^{-3}\right)$ (KÄMPF, 2005). Não foram encontradas referências na literatura do espaço de aeração indicado para cravina de jardim. A indicação para cravos (Dianthus caryophyllus), pertencente ao mesmo gênero da cravina de jardim, é que devem ser cultivados em meios com espaço de aeração entre 2 e 5\% (KÄMPF, 2005). Observa-se que todos os substratos apresentaram valores superiores a esta recomendação.

O substrato S1 tinha $50 \%$ do espaço de aeração do substrato S6, o qual não foi suficiente para garantir o bom desenvolvimento do sistema aéreo, possivelmente em função do sistema radicular ser prejudicado em tais circunstâncias, acarretando em plantas com menor número de ramificações e área foliar e consequentemente com menor fitomassa acumulada. Salvador et al. (2015) estudaram as características físicas adequadas ao crescimento de gloxínia, e acharam que o substrato com densidade de 0,55 $\mathrm{g} \mathrm{cm}^{-3}$ e espaço de aeração de $26,93 \%$ foi o que resultou em maior área foliar. Já Santos et al. (2006) encontraram maior área foliar em mudas de helicônia, quando o substrato apresentou espaço de aeração de $21 \%$. 
Tabela 1. Características químicas e físicas dos substratos utilizados no experimento com cravina de jardim. Table 1. Chemical and physical characteristics of the substrates used in the experiment with chinese pink.

\begin{tabular}{|c|c|c|c|c|c|c|c|c|c|c|c|}
\hline \multicolumn{8}{|c|}{ Características Químicas } & \multicolumn{4}{|c|}{ Características Físicas } \\
\hline \multirow{2}{*}{ Substratos* } & \multirow{2}{*}{$\begin{array}{c}\text { MO } \\
\%\end{array}$} & \multirow{2}{*}{$\begin{array}{c}\text { pH água } \\
1: 1\end{array}$} & $\mathbf{P}$ & $\mathbf{K}$ & $\mathbf{C a}$ & Mg & $\begin{array}{l}\text { CTC } \\
\text { efet. }\end{array}$ & \multirow{2}{*}{$\begin{array}{c}\text { DS } \\
\mathrm{g} \mathrm{cm}^{-3}\end{array}$} & \multirow{3}{*}{$\begin{array}{c}\text { PT } \\
\mathbf{c m}^{3} \mathbf{c m}^{-3} \\
0,56\end{array}$} & \multirow{3}{*}{$\begin{array}{c}\text { AD } \\
\mathbf{c m}^{3} \mathbf{c m}^{-3} \\
0,33\end{array}$} & \multirow{3}{*}{$\begin{array}{c}\text { EA } \\
\mathbf{c m}^{3} \mathbf{c m}^{-3} \\
0,11\end{array}$} \\
\hline & & & \multicolumn{2}{|c|}{$\mathbf{m g ~ d m ^ { - 3 }}$} & \multicolumn{3}{|c|}{$\mathrm{cmol}_{\mathrm{c}} \mathrm{dm}^{-3}$} & & & & \\
\hline $\mathrm{S} 1-\mathrm{S}+\mathrm{C}$ & 0,5 & 6,0 & 83,9 & 440 & 3,5 & 1,0 & 5,6 & 0,57 & & & \\
\hline $\mathrm{S} 2-\mathrm{S}+\mathrm{H}$ & 1,2 & 5,0 & 616,5 & 540 & 5,4 & 2,8 & 11,3 & 0,87 & 0,52 & 0,21 & 0,10 \\
\hline $\mathrm{S} 3-\mathrm{C}+\mathrm{H}$ & 8,6 & 8,7 & 666,7 & 800 & 6,2 & 5,4 & 13,7 & 0,27 & 0,76 & 0,44 & 0,17 \\
\hline $\mathrm{S} 4-\mathrm{S}+\mathrm{C}+\mathrm{H}$ & 2,5 & 6,4 & 138,8 & 800 & 8,3 & 4,2 & 14,6 & 0,62 & 0,63 & 0,29 & 0,17 \\
\hline $\mathrm{S} 5-\mathrm{T}$ & 26,5 & 5,4 & 120,1 & 324 & 64,4 & 6,8 & 72,1 & 0,51 & 0,71 & 0,17 & 0,22 \\
\hline S6-M & 26,5 & 5,7 & 261,4 & 460 & 25,4 & 7,2 & 33,7 & 0,53 & 0,70 & 0,32 & 0,13 \\
\hline $\mathrm{S} 7-\mathrm{T}+\mathrm{C}$ & 26,5 & 6,2 & 550,2 & 800 & 47,6 & 5,8 & 55,5 & 0,36 & 0,70 & 0,32 & 0,13 \\
\hline
\end{tabular}

${ }^{*} \mathrm{~S} 1=50 \%$ solo $+50 \%$ cinza de casca de arroz $(\mathrm{S}+\mathrm{C}), \mathrm{S} 2=80 \%$ solo $+20 \%$ húmus de minhoca $(\mathrm{S}+\mathrm{H}), \mathrm{S} 3=80 \%$ cinzas de casca de arroz $+20 \%$ húmus de minhoca $(\mathrm{C}+\mathrm{H}), \mathrm{S} 4=40 \%$ solo $+40 \%$ cinzas de casca de arroz $+20 \%$ húmus de minhoca $(\mathrm{S}+\mathrm{C}+\mathrm{H}), \mathrm{S} 5=100 \%$ turfa (T), $6=100 \%$ Mecplant $^{\circledR}(\mathrm{M}), \mathrm{S} 7=50 \%$ turfa $+50 \%$ cinzas de casca de $\operatorname{arroz}(\mathrm{T}+\mathrm{C})$.

${ }^{*} \mathrm{~S} 1=50 \%$ soil $+50 \%$ rice husk ash $(S+C) ; S 2=80 \%$ soil $+20 \%$ earthworm castings $(S+H) ; S 3=80 \%$ rice husk ash $+20 \%$ earthworm castings $(C+H) ; S 4=40 \%$ soil $+40 \%$ rice husk ash $+20 \%$ earthworm castings $(S+C+H) ; S 5=100 \%$ peat $(T) ; S 6=100 \%$ commercial substrate Mecplant $^{\circledR}(M) ; S 7=50 \%$ peat $+50 \%$ rice husk ash $(T+C)$.

O cultivo de plantas em recipientes com reduzido volume de substrato leva a uma alta concentração de raízes, exigindo elevado suprimento de oxigênio e rápida remoção do gás carbônico formado. Sendo assim, o substrato deve apresentar porosidade suficiente para permitir trocas gasosas, evitando falta de ar para a respiração das raízes, assim como para a atividade microbiana do meio (KÄMPF, 2005). A porosidade total (PT) ideal para substratos hortícolas é de $0,85 \mathrm{~m}^{3} \mathrm{~m}^{-3}$ (VERDONK e GABRIELS, 1988) e segundo Lopes et al. (2008) o crescimento das mudas é afetado quando a PT não se encontra em níveis ideais, devido à dificuldade de expansão das raízes. Observa-se que nenhum dos substratos atingiu o valor de porosidade total ideal. No entanto, o substrato S3 obteve valor próximo para porosidade total ideal $\left(0,76 \mathrm{~cm}^{3}\right.$ $\mathrm{cm}^{-3}$ ), recomendada pela literatura.

Considerando-se os limites das características físicas acima, observou-se que todos os substratos apresentam algum fator limitante. No entanto, essas faixas ideais podem ser variáveis, para cada experimento, pois se deve considerar o material utilizado, as condições de cultivo, o manejo, dentre outros.
Em relação às propriedades químicas (Tabela 1), pelos resultados de $\mathrm{pH}$, os substratos $\mathrm{S} 1, \mathrm{~S} 4, \mathrm{~S} 5$ e S6 apresentaram valores preconizados para suas respectivas composições, ou seja, nos substratos com predominância de matéria orgânica (MO), a faixa de $\mathrm{pH}$ recomendada é de 5,0 a 5,8; quando for a base de solo mineral, entre 6,0 e 6,5, além disso, problemas com a disponibilidade de fósforo $(\mathrm{P})$, são esperados em pH acima de 6,5 (KÄMPF, 2005), como é o caso do substrato S3. Os substratos S5, S6 e S7, com 26,5\% de MO, apresentaram os maiores teores de $\mathrm{Ca}$ e $\mathrm{Mg}$ (Tabela 1). A matéria orgânica costuma alterar o complexo coloidal, criar cargas superficiais e, consequentemente, aumentar a CTC, favorecendo a alta quantidade de nutrientes disponíveis para a planta (BEZERRA et al., 2006).

$\mathrm{Na}$ análise sobre a aderência à distribuição normal das variáveis analisadas por substrato, o substrato $\mathrm{S} 1$, teve $82 \%$ dos variáveis avaliados sem distribuição normal de probabilidade, com exceção, aos variáveis: diâmetro da haste principal a 5 e $10 \mathrm{~cm}$ da base do substrato e fitomassa seca total da parte aérea. Enquanto que, o substrato S2 apresentou $71 \%$ de variáveis com distribuição normal (Tabela 2). 
Tabela 2. Ajuste à distribuição normal de probabilidade para diferentes substratos (S1 a S7) e variáveis avaliados no cultivo de cravina de jardim.

Table 2. Set to normal probability distribution for different substrates (S1 to S7) and traits in the cultivation of chinese pink.

\begin{tabular}{|c|c|c|c|c|c|c|c|}
\hline \multirow{2}{*}{ Variáveis } & \multicolumn{7}{|c|}{ Substratos* } \\
\hline & S1 & S2 & S3 & S4 & S5 & S6 & S7 \\
\hline Número de ramificações & $\mathrm{N}^{* *}$ & $\mathrm{~N}$ & $\mathrm{~S}$ & $\mathrm{~N}$ & $\mathrm{~N}$ & $\mathrm{~N}$ & $\mathrm{~N}$ \\
\hline Número de botões florais & $\mathrm{N}$ & $\mathrm{S}$ & $\mathrm{N}$ & $\mathrm{N}$ & $\mathrm{N}$ & $\mathrm{S}$ & $\mathrm{N}$ \\
\hline Área foliar haste principal $\left(\mathrm{cm}^{2}\right)$ & $\mathrm{N}$ & $\mathrm{S}$ & $\mathrm{S}$ & $\mathrm{N}$ & $\mathrm{S}$ & $\mathrm{S}$ & $\mathrm{S}$ \\
\hline Área foliar ramificações $\left(\mathrm{cm}^{2}\right)$ & $\mathrm{N}$ & $\mathrm{S}$ & $\mathrm{S}$ & $\mathrm{S}$ & $\mathrm{N}$ & $\mathrm{S}$ & $\mathrm{N}$ \\
\hline Área foliar total parte aérea $\left(\mathrm{cm}^{2}\right)$ & $\mathrm{N}$ & $\mathrm{S}$ & $\mathrm{S}$ & $\mathrm{S}$ & $\mathrm{S}$ & $\mathrm{S}$ & $\mathrm{S}$ \\
\hline Ciclo até a diferenciação (dias) & $\mathrm{N}$ & $\mathrm{N}$ & $\mathrm{N}$ & $\mathrm{N}$ & $\mathrm{N}$ & $\mathrm{N}$ & $\mathrm{N}$ \\
\hline Ciclo até o aparecimento do $1^{\circ}$ botão floral (dias) & $\mathrm{N}$ & $\mathrm{N}$ & $\mathrm{N}$ & $\mathrm{N}$ & $\mathrm{N}$ & $\mathrm{N}$ & $\mathrm{N}$ \\
\hline Ciclo de produção (dias) & $\mathrm{N}$ & $\mathrm{N}$ & $\mathrm{N}$ & $\mathrm{N}$ & $\mathrm{N}$ & $\mathrm{N}$ & $\mathrm{N}$ \\
\hline Diâmetro (D.) da flor (cm) & $\mathrm{N}$ & $\mathrm{N}$ & $\mathrm{N}$ & $\mathrm{S}$ & $\mathrm{N}$ & $\mathrm{N}$ & $\mathrm{N}$ \\
\hline D. da haste principal a $5 \mathrm{~cm}$ da base $(\mathrm{mm})$ & $\mathrm{S}$ & $\mathrm{S}$ & $\mathrm{S}$ & $\mathrm{S}$ & $\mathrm{S}$ & $\mathrm{S}$ & $\mathrm{S}$ \\
\hline D. da haste principal a $10 \mathrm{~cm}$ da base $(\mathrm{mm})$ & $\mathrm{S}$ & $\mathrm{S}$ & $\mathrm{S}$ & $\mathrm{S}$ & $\mathrm{S}$ & $\mathrm{N}$ & $\mathrm{S}$ \\
\hline Fitomassa verde haste principal (g) & $\mathrm{N}$ & $\mathrm{S}$ & $\mathrm{S}$ & $\mathrm{S}$ & $\mathrm{N}$ & $\mathrm{S}$ & $\mathrm{S}$ \\
\hline Fitomassa verde ramificações (g) & $\mathrm{N}$ & $\mathrm{S}$ & $\mathrm{S}$ & $\mathrm{S}$ & $\mathrm{S}$ & $\mathrm{N}$ & $\mathrm{N}$ \\
\hline Fitomassa verde total parte aérea $(\mathrm{g})$ & $\mathrm{N}$ & $\mathrm{S}$ & $\mathrm{S}$ & $\mathrm{S}$ & $\mathrm{N}$ & $\mathrm{N}$ & $\mathrm{N}$ \\
\hline Fitomassa seca haste principal (g) & $\mathrm{N}$ & $\mathrm{S}$ & $\mathrm{N}$ & $\mathrm{S}$ & $\mathrm{S}$ & $\mathrm{S}$ & $\mathrm{S}$ \\
\hline Fitomassa seca ramificações (g) & $\mathrm{N}$ & $\mathrm{S}$ & $\mathrm{S}$ & $\mathrm{S}$ & $\mathrm{S}$ & $\mathrm{N}$ & $\mathrm{N}$ \\
\hline Fitomassa seca total parte aérea $(\mathrm{g})$ & $\mathrm{S}$ & $\mathrm{S}$ & $\mathrm{S}$ & $\mathrm{S}$ & $\mathrm{S}$ & $\mathrm{N}$ & $\mathrm{N}$ \\
\hline
\end{tabular}

${ }^{*} \mathrm{~S} 1=50 \%$ solo $+50 \%$ cinza de casca de arroz, $\mathrm{S} 2=80 \%$ solo $+20 \%$ húmus de minhoca, $\mathrm{S} 3=80 \%$ cinzas de casca de arroz $+20 \%$ húmus de minhoca, S4=40\% solo $+40 \%$ cinzas de casca de arroz $+20 \%$ húmus de minhoca, S5 $=100 \%$ turfa, S6=100\% Mecplant ${ }^{\mathbb{R}}, \mathrm{S} 7=50 \%$ turfa $+50 \%$ cinzas de casca de arroz. ${ }^{* *}$ Não ajuste a distribuição normal (N) e ajuste a distribuição normal de probabilidade (S), pelo teste de Anderson-Darling (valor-p $>0,05$ ).

${ }^{*} \mathrm{~S} 1=50 \%$ soil $+50 \%$ rice husk ash, $S 2=80 \%$ soil $+20 \%$ earthworm vermicompost, $S 3=80 \%$ rice husk ash $+20 \%$ earthworm vermicompost, $S 4=40 \%$ soil $+40 \%$ rice husk ash $+20 \%$ earthworm vermicompost, $S 5=100 \%$ peat, $S 6=100 \%$ commercial substrate Mecplant $^{\circledR}, S 7=50 \%$ peat $+50 \%$ rice husk ash. ${ }^{* *}$ Do not adjust the normal distribution $(N)$ and set the normal distribution $(S)$, the Anderson-Darling test (p-value $>0.05$ ).

Caracterizando os dados pelos coeficientes de variação (Tabela 3), o substrato S1 foi o que apresentou o maior número de variáveis (cinco) em que o desvio-padrão supera em mais de 50\% do valor da média, proporcionando maior variabilidade dos dados do que os demais substratos. Este aumento na variabilidade entre plantas pode ser explicado pela variabilidade do próprio material utilizado na composição deste substrato: cinzas de casca de arroz. Provavelmente, este material apresentou variabilidade na sua elaboração, no armazenamento no engenho e no momento de coleta, o que causou heterogeneidade e proporcionou fonte de variação entre as plantas cultivadas neste substrato, acarretando em erro experimental. Diversas fontes de erro experimental estão presentes em experimentos, e aqueles conduzidos em ambiente protegido também são afetados por essas fontes de heterogeneidade, entre elas a heterogeneidade do material experimental utilizado (LORENTZ et al., 2004, 2005). 
Tabela 3. Ajuste a distribuição normal de probabilidade (D) e teste de homogeneidade das variâncias (HV) de 392 plantas, e coeficiente de variação para diferentes substratos (S1 a S7) em 17 variáveis de cravina de jardim.

Table 3. Set the normal distribution (D) and variances homogeneity test (HV) of 392 plants, and coefficient of variation for different substrates (S1 to S7) in 17 characters of chinese pink

\begin{tabular}{|c|c|c|c|c|c|c|c|c|c|}
\hline & \multicolumn{9}{|c|}{ Coeficiente de variação (\%) } \\
\hline Variáveis $^{* *}$ & $\mathbf{D}^{\#}$ & $\mathbf{H V}$ & $\mathbf{S 1}^{*}$ & S2 & S3 & S4 & S5 & S6 & S7 \\
\hline NR & $\mathrm{N}$ & NH & 88,86 & 12,85 & 18,18 & 13,45 & 16,78 & 11,00 & 19,76 \\
\hline NBF & $\mathrm{N}$ & $\mathrm{NH}$ & 76,20 & 36,05 & 54,86 & 46,01 & 58,36 & 34,17 & 67,27 \\
\hline $\operatorname{AFHP}\left(\mathrm{cm}^{2}\right)$ & $\mathrm{N}$ & $\mathrm{NH}$ & 26,91 & 12,00 & 41,97 & 16,77 & 11,14 & 16,64 & 19,92 \\
\hline $\operatorname{AFR}\left(\mathrm{cm}^{2}\right)$ & $\mathrm{N}$ & NH & 126,72 & 25,99 & 37,62 & 21,06 & 27,90 & 26,36 & 48,10 \\
\hline AFT $\left(\mathrm{cm}^{2}\right)$ & $\mathrm{N}$ & NH & 39,34 & 17,47 & 27,65 & 15,17 & 16,81 & 21,25 & 31,66 \\
\hline $\mathrm{CD}$ (dias) & $\mathrm{N}$ & NH & 23,28 & 9,64 & 19,40 & 7,69 & 9,22 & 8,86 & 9,26 \\
\hline $\mathrm{CB}$ (dias) & $\mathrm{N}$ & NH & 21,41 & 7,58 & 14,31 & 7,21 & 8,21 & 7,25 & 9,34 \\
\hline $\mathrm{CP}$ (dias) & $\mathrm{N}$ & NH & 17,17 & 7,74 & 15,94 & 6,71 & 7,64 & 6,58 & 7,85 \\
\hline $\mathrm{DF}(\mathrm{cm})$ & $\mathrm{N}$ & $\mathrm{NH}$ & 6,82 & 6,72 & 16,46 & 7,68 & 8,83 & 6,65 & 9,44 \\
\hline DHP a $5 \mathrm{~cm}(\mathrm{~mm})$ & $\mathrm{S}$ & $+\mathrm{NH}$ & 22,26 & 9,47 & 13,93 & 11,71 & 10,31 & 8,89 & 10,08 \\
\hline DHP a $10 \mathrm{~cm}(\mathrm{~mm})$ & $\mathrm{S}$ & $+\mathrm{NH}$ & 18,30 & 11,95 & 16,56 & 11,28 & 12,55 & 11,84 & 17,81 \\
\hline FVHP (g) & $\mathrm{N}$ & NH & 28,07 & 12,34 & 30,21 & 11,78 & 13,09 & 13,96 & 18,10 \\
\hline FVR (g) & $\mathrm{N}$ & NH & 117,94 & 22,78 & 36,87 & 21,88 & 26,46 & 19,94 & 46,18 \\
\hline FVT (g) & $\mathrm{N}$ & NH & 37,85 & 14,32 & 20,79 & 14,53 & 15,08 & 15,46 & 28,94 \\
\hline FSHP $(g)$ & $\mathrm{S}$ & $+\mathrm{NH}$ & 26,73 & 13,27 & 27,62 & 13,35 & 10,56 & 16,70 & 19,19 \\
\hline FSR (g) & $\mathrm{N}$ & NH & 120,93 & 25,02 & 38,15 & 25,80 & 30,82 & 30,48 & 60,46 \\
\hline \multirow[t]{2}{*}{ FST (g) } & $\mathrm{N}$ & $\mathrm{NH}$ & 31,03 & 16,58 & 23,73 & 17,36 & 17,62 & 25,73 & 36,86 \\
\hline & & Média & 48,81 & 15,40 & 26,73 & 15,85 & 17,73 & 16,57 & 27,07 \\
\hline
\end{tabular}

${ }^{*} \mathrm{~S} 1=50 \%$ solo $+50 \%$ cinza de casca de arroz, $\mathrm{S} 2=80 \%$ solo $+20 \%$ húmus de minhoca, $\mathrm{S} 3=80 \%$ cinzas de casca de arroz $+20 \%$ húmus de minhoca, S4 $=40 \%$ solo $+40 \%$ cinzas de casca de arroz $+20 \%$ húmus de minhoca, S5 $=100 \%$ turfa, S6 $=100 \%$ Mecplant ${ }^{\circledR}, \mathrm{S} 7=50 \%$ turfa $+50 \%$ cinzas de casca de arroz. ${ }^{* *} \mathrm{NR}=$ número de ramificações; $\mathrm{NBF}=$ número de botões florais; $\mathrm{AFHP}=$ área foliar da haste principal; $\mathrm{AFR}=$ área foliar das ramificações; $\mathrm{AFT}=$ área foliar total da parte aérea; $\mathrm{CD}=$ ciclo até a diferenciação (dias após a repicagem - $\mathrm{DAR}$ ); $\mathrm{CB}=$ ciclo até o aparecimento do primeiro botão floral (DAR); $\mathrm{CP}=$ ciclo de produção (DAR); $\mathrm{DF}=$ diâmetro de flor; $\mathrm{DHP}$ a $5 \mathrm{~cm}=\mathrm{Diâmetro}$ da haste principal a $5 \mathrm{~cm}$ da base; DHP a $10 \mathrm{~cm}=$ Diâmetro da haste principal a $10 \mathrm{~cm}$ da base; $\mathrm{FVHP}=$ fitomassa verde da haste principal; FVR=fitomassa verde das ramificações; FVT=fitomassa verde total parte aérea; FSHP=fitomassa seca da haste principal; FSR=fitomassa seca das ramificações; FST=fitomassa seca total parte aérea. " Distribuição de probabilidade normal (S) não normal (N), pelo teste de Anderson-Darling (valor-p>0,05) "\#ariância não homogênea pelo teste de Levene $(\mathrm{NH})$ e pelo teste de Bartlet $(+\mathrm{NH})(\mathrm{valor}-\mathrm{p}>0,05)$. ${ }^{*} \mathrm{~S} 1=50 \%$ soil $+50 \%$ rice husk ash, $S 2=80 \%$ soil $+20 \%$ earthworm vermicompost, $S 3=80 \%$ rice husk ash $+20 \%$ earthworm vermicompost, $S 4=40 \%$ soil $+40 \%$ rice husk ash $+20 \%$ earthworm vermicompost, $S 5=100 \%$ peat, $S 6=100 \%$ commercial substrate Mecplant $^{\mathbb{B}}, S 7=50 \%$ peat $+50 \%$ rice husk ash ${ }^{* *} N R=$ number of branches; $N B F=$ number of flower buds; $A F H P=$ leaf area of the main stem; $A F R=$ leaf area of the branches; $A F T=$ total leaf area of shoots; $C D=$ cycle to the differentiation (days after transplanting - DAT); $C B=$ cycle until the appearance of the first flower bud (DAT); $C P=$ production cycle (DAT); DF=flower diameter; DHP of $5 \mathrm{~cm}=$ main stem diameter of $5 \mathrm{~cm}$ from the base; DHP of $10 \mathrm{~cm}=$ main stem diameter of $10 \mathrm{~cm}$ from the base; FVHP=green biomass main stem; $F V R=$ green biomass of branches; FVT=total green biomass of shoots; FSHP=dry mass of the main stem; FSR=dry mass of branches; FST $=$ total dry mass of the shoots. "Normal probability distribution (S) not normal (N), the Anderson-Darling test (p-value $>0.05)$. \#Inhomogeneous variance by Levene test $(\mathrm{NH})$ and the Bartlet test $(+\mathrm{NH})(p$-value $>0.05)$. 
Das 17 variáveis avaliadas, aproximadamente, 25\% delas não tiveram influência do tipo de substrato, ao atender ou não a normalidade dos dados: em todos os substratos, as variáveis ciclo até a diferenciação, ciclo até o aparecimento do primeiro botão floral e ciclo de produção, não se ajustaram à distribuição normal; e, a variável diâmetro da haste principal a $5 \mathrm{~cm}$ foi o único que se ajustou à distribuição normal em todos os substratos (Tabela 2). Para a variável diâmetro da haste principal a $5 \mathrm{~cm}$, o desviopadrão não ultrapassa em 13\%, em média dos substratos, do valor da média (Tabela 3). Portanto, a forma de obtenção da variável, por contagem, é mais importante na influência da normalidade dos dados do que o tipo de substrato.

Além do ciclo, as outras variáveis obtidas por contagem não se ajustaram à distribuição normal de probabilidade na maioria dos substratos utilizados, como foi o caso do número de ramificações e de botões (Tabela 2). O substrato com $50 \%$ solo $+50 \%$ cinzas de casca de arroz apresentou, para número de ramificações, excesso de valores zeros $(39 \%$ dos dados), o que pode contribuir para o não atendimento à normalidade dos erros (LÚCIO et al., 2010). Isso pode ser explicado pela composição das cinzas de casca de arroz carbonizada que são compostas basicamente de sílica e, portanto, bastante alcalinas (DEY et al., 2013; SARANGI et al., 2011). Um pH muito elevado ou elevada alcalinidade, acima de 6,5, diminui demasiadamente a disponibilidade de fósforo e micronutrientes (cobre, zinco, ferro e manganês) às plantas (KAMPF, 2005) e estas sob deficiência de $\mathrm{P}$ apresentam atraso no florescimento (MALAVOLTA, 2006). Este atraso no florescimento é reflexo do menor número de ramificações na planta, pois à medida que vão aparecendo ramificações na planta é porque já existe uma área foliar mínima que garante a produção de fotoassimilados para o florescimento (FAGUNDES et al., 2008).

Testando o ajuste à distribuição normal de todos os dados, por variável, sem considerar os substratos, três se ajustaram à distribuição normal de probabilidade: diâmetro da haste principal a 5 e $10 \mathrm{~cm}$ e fitomassa seca da haste principal (Tabela 3). Desses três, o diâmetro da haste principal a $5 \mathrm{~cm}$ foi a única variável que teve ajuste da normalidade por substrato (Tabela 2) e de forma geral, considerando somente a variável, ou seja, a distribuição normal dos dados dessa variável independe dos substratos. Resultados com hortaliças de múltiplas colheitas indicam a violação da pressuposição de normalidade dos erros, tanto para variáveis produtivos quanto morfológicos, bem como, quando obtidas em tratamentos diferentes (LUCIO et al., 2012).

Os coeficientes de variação médios (Tabela 3) das variáveis, por substrato, indicam maior variabilidade no substrato S1 $(48,81 \%)$, que se explica, em parte, pelo excesso de valores zeros do número de ramificações, e por conseguinte em: área foliar das ramificações, fitomassa verde das ramificações e fitomassa seca das ramificações, fato este que, também, havia influenciado no não atendimento à normalidade na maioria das variáveis deste substrato.

As menores variabilidades encontradas nos substratos S2, S4, S5 e S6, com coeficientes de variação médio, aproximado, de 16\%, implicaram em heterogeneidade de variâncias dos substratos, pelo teste de Bartlett/Levene, para todas as variáveis (Tabela 3). Exceto os substratos S5 e S6, os demais foram obtidos de misturas de componentes, o que, possivelmente, proporcione maior variabilidade entre as unidades experimentais do mesmo substrato (saco com uma planta) do que entre as unidades experimentais dos substratos com somente um componente (substrato S5, com turfa, e substrato S6, com Mecplant ${ }^{\circledR}$ ).

Diversas fontes de erro experimental estão presentes em experimentos, e a heterogeneidade do material experimental deve ser minimizada, pois aumenta o quadrado médio do erro, e consequentemente, diminui a estatística $\mathrm{F}$ numa análise de variância, com maior probabilidade de aceitar a hipótese de igualdade de contrastes entre médias de tratamento. Além disso, pode acarretar em não atendimento da pressuposição de homogeneidade das variâncias dos erros, o que interfere na estimativa do erro experimental, com influência direta nos testes de hipótese.

Em função da maioria dos variáveis não apresentar distribuição normal, foi utilizado para determinar o tamanho de amostra (número de plantas) neste trabalho, a metodologia de reamostragem com reposição, que é o indicado nestes casos, pois independe da distribuição de probabilidade de cada variável (FERREIRA, 2009).

Observa-se variação no tamanho de amostra para cada substrato utilizado e variável avaliada, para uma amplitude do intervalo de confiança de $95 \%$ da média (Tabelas 4 e 5). Este tamanho, para uma amplitude do intervalo de confiança (IC $=95 \%$ ) igual a $5 \%$ da média (D5), oscilou de 24 plantas, no substrato comercial Mecplant ${ }^{\mathbb{B}}$, para a variável ciclo de produção, até acima de 300 plantas nos diferentes substratos e variáveis avaliadas. Isto é explicado pois, o tamanho de amostra é diretamente proporcional à variabilidade dos dados, que depende da homogeneidade dos substratos utilizados e da variável avaliada.

A variabilidade de tamanho de amostra entre variáveis e tratamentos, também já foi constatada com genótipos de milho (STORCK et al., 2007), de feijão (CARGNELUTTI FILHO et al., 2008) e variáveis de produção de mamona (CARGNELUTTI FILHO et al., 2010a). 
Tabela 4. Tamanho de amostra (número de plantas) para uma amplitude do intervalo de confiança ( $\mathrm{p}=95 \%$ ) igual a 5\% (D5), 10\% (D10), 20\% (D20) e 40\% (D40) da média em diferentes variáveis de cravina de jardim, cultivada com sete substratos. Table 4. Sample size (number of plants) for a confidence interval width (w=95\%) at $5 \%$ (D5), $10 \%$ (D10), 20\% (D20) and $40 \%$ (D4) of the average at different character cravina garden, cultivated with seven substrates.

\begin{tabular}{|c|c|c|c|c|c|c|c|c|c|c|c|c|}
\hline \multirow{2}{*}{ Substratos" } & \multicolumn{4}{|c|}{ Número de ramificações } & \multicolumn{4}{|c|}{ Número de botões florais } & \multicolumn{4}{|c|}{$\begin{array}{l}\text { Área foliar da haste principal } \\
\qquad\left(\mathrm{cm}^{2}\right)\end{array}$} \\
\hline & D5 & D10 & D20 & D40 & D5 & D10 & D20 & D40 & D5 & D10 & D20 & D40 \\
\hline $\mathrm{S} 1-\mathrm{S}+\mathrm{C}$ & 300 & 99 & 285 & 72 & 300 & 299 & 212 & 52 & 300 & 108 & 29 & 7 \\
\hline $\mathrm{S} 2-\mathrm{S}+\mathrm{H}$ & 97 & 23 & 6 & 2 & 300 & 195 & 50 & 12 & 87 & 23 & 6 & 2 \\
\hline $\mathrm{S} 3-\mathrm{C}+\mathrm{H}$ & 191 & 51 & 12 & 4 & 300 & 299 & 113 & 29 & 300 & 257 & 60 & 17 \\
\hline $\mathrm{S} 4-\mathrm{S}+\mathrm{C}+\mathrm{H}$ & 106 & 27 & 8 & 2 & 300 & 299 & 78 & 21 & 163 & 42 & 12 & 3 \\
\hline S5 - T & 165 & 41 & 11 & 3 & 300 & 299 & 128 & 32 & 75 & 20 & 5 & 2 \\
\hline S6 - M & 71 & 17 & 5 & 2 & 300 & 166 & 44 & 11 & 163 & 39 & 11 & 3 \\
\hline $\mathrm{S} 7-\mathrm{T}+\mathrm{C}$ & 220 & 62 & 15 & 4 & 300 & 299 & 164 & 43 & 225 & 58 & 15 & 4 \\
\hline \multirow{2}{*}{ Substratos" } & \multicolumn{4}{|c|}{ Área foliar das ramificações $\left(\mathrm{cm}^{2}\right)$} & \multicolumn{4}{|c|}{$\begin{array}{l}\text { Área foliar total parte aérea } \\
\qquad\left(\mathrm{cm}^{2}\right)\end{array}$} & \multicolumn{4}{|c|}{ Ciclo até a diferenciação (dias) ${ }^{* *}$} \\
\hline & D5 & D10 & D20 & D40 & D5 & D10 & D20 & D40 & D5 & D10 & D20 & D40 \\
\hline $\mathrm{S} 1-\mathrm{S}+\mathrm{C}$ & 300 & 299 & 298 & 144 & 300 & 223 & 56 & 15 & 300 & 81 & 20 & 5 \\
\hline $\mathrm{S} 2-\mathrm{S}+\mathrm{H}$ & 300 & 102 & 25 & 7 & 173 & 46 & 12 & 3 & 51 & 15 & 4 & 2 \\
\hline $\mathrm{S} 3-\mathrm{C}+\mathrm{H}$ & 300 & 212 & 54 & 14 & 300 & 110 & 29 & 8 & 221 & 56 & 14 & 4 \\
\hline $\mathrm{S} 4-\mathrm{S}+\mathrm{C}+\mathrm{H}$ & 260 & 69 & 17 & 4 & 132 & 34 & 9 & 3 & 36 & 9 & 3 & 2 \\
\hline S5 - T & 300 & 112 & 31 & 8 & 164 & 42 & 11 & 3 & 52 & 14 & 3 & 2 \\
\hline S6 - M & 300 & 101 & 26 & 7 & 268 & 66 & 18 & 5 & 47 & 13 & 4 & 2 \\
\hline $\mathrm{S} 7-\mathrm{T}+\mathrm{C}$ & 300 & 299 & 87 & 21 & 300 & 150 & 37 & 10 & 50 & 14 & 4 & 2 \\
\hline \multirow[t]{2}{*}{ Substratos* } & \multicolumn{4}{|c|}{$\begin{array}{l}\text { Ciclo até o aparecimento do } 1^{0} \\
\text { botão floral (dias) }\end{array}$} & \multicolumn{4}{|c|}{ Ciclo de produção (dias) } & & & & \\
\hline & D5 & D10 & D20 & D40 & D5 & D10 & D20 & D40 & & & & \\
\hline $\mathrm{S} 1-\mathrm{S}+\mathrm{C}$ & 255 & 69 & 17 & 4 & 176 & 45 & 12 & 3 & & & & \\
\hline $\mathrm{S} 2-\mathrm{S}+\mathrm{H}$ & 34 & 9 & 3 & 2 & 38 & 9 & 3 & 2 & & & & \\
\hline $\mathrm{S} 3-\mathrm{C}+\mathrm{H}$ & 119 & 31 & 8 & 3 & 157 & 37 & 10 & 3 & & & & \\
\hline $\mathrm{S} 4-\mathrm{S}+\mathrm{C}+\mathrm{H}$ & 33 & 8 & 3 & 2 & 28 & 8 & 3 & 2 & & & & \\
\hline $\mathrm{S} 5-\mathrm{T}$ & 40 & 10 & 3 & 2 & 36 & 9 & 3 & 2 & & & & \\
\hline S6 - M & 30 & 8 & 3 & 2 & 24 & 7 & 3 & 2 & & & & \\
\hline $\mathrm{S} 7-\mathrm{T}+\mathrm{C}$ & 56 & 14 & 4 & 2 & 38 & 10 & 3 & 2 & & & & \\
\hline
\end{tabular}

${ }^{*} \mathrm{~S} 1=50 \%$ solo $+50 \%$ cinza de casca de arroz; $\mathrm{S} 2=80 \%$ solo $+20 \%$ húmus de minhoca; $\mathrm{S} 3=80 \%$ cinzas de casca de arroz $+20 \%$ húmus de minhoca; S4=40\% solo $+40 \%$ cinzas de casca de arroz $+20 \%$ húmus de minhoca; S5 $=100 \%$ turfa; S6=100\% Mecplant ${ }^{\circledR} ; \mathrm{S} 7=50 \%$ turfa $+50 \%$ cinzas de casca de arroz. ${ }^{* *}$ Substrato $\mathrm{S}+\mathrm{C}(\mathrm{N}=48), \mathrm{C}+\mathrm{H}(\mathrm{N}=52)$ e demais $(\mathrm{N}=56)$.

${ }^{*} \mathrm{~S} 1=50 \%$ soil $+50 \%$ rice husk ash, $S 2=80 \%$ soil $+20 \%$ earthworm vermicompost, $S 3=80 \%$ rice husk ash $+20 \%$ earthworm vermicompost, $S 4=40 \%$ soil $+40 \%$ rice husk ash $+20 \%$ earthworm vermicompost, $S 5=100 \%$ peat, S6 $=100 \%$ commercial substrate Mecplant $^{\mathbb{B}}, S 7=50 \%$ peat $+50 \%$ rice husk ash. ${ }^{* *}$ Substrate $S+C(N=48), C+H(N=52)$ and other $(N=56)$. 
Outro fator que influencia o tamanho de amostra é a fertilidade do solo. Resende e Souza Junior (1997) determinaram números de plantas de milho diferentes entre uma condição de solo fértil e outra de cerrado, com maior precisão no solo fértil para a variável produtiva. Considerando o substrato $\mathrm{S} 1$, com os menores valores de $\mathrm{MO} \%, \mathrm{P}, \mathrm{Ca}, \mathrm{Mg}$ e CTC, do que os demais substratos (Tabela 1), observa-se nas Tabelas 4 e 5, que o tamanho de amostra, normalmente, é maior nesse substrato, em qualquer das simulações de precisão simuladas (D5, D10, D20 e D40) e variáveis avaliadas. Os resultados evidenciam ainda, que o tamanho de amostra da variável produtiva, número de botões florais por planta, é maior (em média, 113 plantas, para D20) do que para as variáveis vegetativas. Resultados semelhantes foram obtidos em mamona (CARGNELUTTI FILHO et al., 2010a) e crambe (CARGNELUTTI FILHO et al., 2010b).

Tabela 5. Tamanho de amostra (número de plantas) para uma amplitude do intervalo de confiança ( $\mathrm{p}=95 \%$ ) igual a 5\% (D5), 10\% (D10), 20\% (D20) e 40\% (D40) da média em diferentes variáveis de cravina de jardim, cultivada com sete substratos. Table 5. Sample size (number of plants) for a confidence interval width (w=95\%) at $5 \%$ (D5), $10 \%$ (D10), $20 \%$ (D20) and $40 \%$ (D40) of the average at different character cravina garden, cultivated with seven substrates.

\begin{tabular}{|c|c|c|c|c|c|c|c|c|c|c|c|c|}
\hline \multirow[t]{2}{*}{ Substratos ${ }^{*}$} & \multicolumn{4}{|c|}{ Diâmetro da flor $(\mathrm{cm})^{* * *}$} & \multicolumn{4}{|c|}{$\begin{array}{c}\text { Diâmetro da haste principal a } \\
5 \mathrm{~cm} \text { da base }(\mathrm{mm})^{* * *}\end{array}$} & \multicolumn{4}{|c|}{$\begin{array}{l}\text { Diâmetro da haste principal a } \\
10 \mathrm{~cm} \text { da base }(\mathrm{mm})^{* * *}\end{array}$} \\
\hline & D5 & D10 & D20 & D40 & D5 & D10 & D20 & D40 & D5 & D10 & D20 & D40 \\
\hline $\mathrm{S} 1-\mathrm{S}+\mathrm{C}$ & 28 & 8 & 3 & 2 & 283 & 74 & 19 & 5 & 193 & 48 & 12 & 3 \\
\hline $\mathrm{S} 2-\mathrm{S}+\mathrm{H}$ & 27 & 7 & 3 & 2 & 51 & 14 & 4 & 2 & 86 & 22 & 6 & 2 \\
\hline $\mathrm{S} 3-\mathrm{C}+\mathrm{H}$ & 154 & 39 & 11 & 3 & 116 & 30 & 8 & 2 & 160 & 42 & 11 & 3 \\
\hline $\mathrm{S} 4-\mathrm{S}+\mathrm{C}+\mathrm{H}$ & 37 & 9 & 3 & 2 & 81 & 22 & 6 & 2 & 76 & 19 & 5 & 2 \\
\hline S5 - T & 48 & 13 & 4 & 2 & 65 & 16 & 5 & 2 & 96 & 23 & 7 & 2 \\
\hline S6 - M & 27 & 7 & 3 & 2 & 46 & 12 & 4 & 2 & 83 & 21 & 6 & 2 \\
\hline $\mathrm{S} 7-\mathrm{T}+\mathrm{C}$ & 53 & 14 & 4 & 2 & 60 & 15 & 4 & 2 & 189 & 47 & 12 & 3 \\
\hline \multirow[t]{2}{*}{ Substratos ${ }^{*}$} & \multicolumn{4}{|c|}{$\begin{array}{c}\text { Fitomassa verde da haste } \\
\text { principal (g) }\end{array}$} & \multicolumn{4}{|c|}{$\begin{array}{l}\text { Fitomassa verde das } \\
\text { ramificações }(\mathrm{g})\end{array}$} & \multicolumn{4}{|c|}{$\begin{array}{c}\text { Fitomassa verde total da parte } \\
\text { aérea (g) }\end{array}$} \\
\hline & D5 & D10 & D20 & D40 & D5 & D10 & D20 & D40 & D5 & D10 & D20 & D40 \\
\hline $\mathrm{S} 1-\mathrm{S}+\mathrm{C}$ & 300 & 116 & 30 & 8 & 300 & 299 & 298 & 127 & 300 & 212 & 53 & 14 \\
\hline $\mathrm{S} 2-\mathrm{S}+\mathrm{H}$ & 89 & 23 & 6 & 2 & 298 & 80 & 19 & 6 & 121 & 31 & 8 & 2 \\
\hline $\mathrm{S} 3-\mathrm{C}+\mathrm{H}$ & 300 & 132 & 34 & 9 & 300 & 202 & 51 & 13 & 249 & 64 & 17 & 5 \\
\hline $\mathrm{S} 4-\mathrm{S}+\mathrm{C}+\mathrm{H}$ & 79 & 22 & 6 & 2 & 289 & 71 & 17 & 5 & 128 & 33 & 9 & 3 \\
\hline S5 - T & 90 & 26 & 8 & 3 & 300 & 103 & 27 & 7 & 133 & 35 & 9 & 3 \\
\hline S6 - M & 118 & 30 & 8 & 2 & 239 & 62 & 15 & 4 & 142 & 35 & 9 & 3 \\
\hline $\mathrm{S} 7-\mathrm{T}+\mathrm{C}$ & 180 & 48 & 12 & 4 & 300 & 299 & 81 & 20 & 300 & 122 & 30 & 9 \\
\hline \multirow[t]{2}{*}{ Substratos ${ }^{*}$} & \multicolumn{4}{|c|}{$\begin{array}{c}\text { Fitomassa seca da haste } \\
\text { principal (g) }\end{array}$} & \multicolumn{4}{|c|}{$\begin{array}{c}\text { Fitomassa seca das } \\
\text { ramificações }(\mathrm{g})\end{array}$} & \multicolumn{4}{|c|}{$\begin{array}{c}\text { Fitomassa seca total da parte } \\
\text { aérea }(g)\end{array}$} \\
\hline & D5 & D10 & D20 & D40 & D5 & D10 & D20 & D40 & D5 & D10 & D20 & D40 \\
\hline $\mathrm{S} 1-\mathrm{S}+\mathrm{C}$ & 300 & 106 & 26 & 7 & 300 & 299 & 298 & 133 & 300 & 138 & 36 & 10 \\
\hline $\mathrm{S} 2-\mathrm{S}+\mathrm{H}$ & 105 & 26 & 7 & 2 & 300 & 90 & 25 & 6 & 162 & 41 & 11 & 3 \\
\hline $\mathrm{S} 3-\mathrm{C}+\mathrm{H}$ & 300 & 111 & 28 & 7 & 300 & 218 & 56 & 14 & 300 & 84 & 21 & 6 \\
\hline $\mathrm{S} 4-\mathrm{S}+\mathrm{C}+\mathrm{H}$ & 106 & 26 & 7 & 2 & 300 & 96 & 27 & 6 & 168 & 46 & 12 & 3 \\
\hline $\mathrm{S} 5-\mathrm{T}$ & 65 & 17 & 5 & 2 & 300 & 142 & 37 & 9 & 182 & 45 & 12 & 3 \\
\hline S6 - M & 162 & 42 & 11 & 3 & 300 & 135 & 35 & 9 & 300 & 99 & 25 & 7 \\
\hline $\mathrm{S} 7-\mathrm{T}+\mathrm{C}$ & 213 & 54 & 14 & 4 & 300 & 299 & 129 & 35 & 300 & 204 & 50 & 13 \\
\hline
\end{tabular}

${ }^{*} \mathrm{~S} 1=50 \%$ solo $+50 \%$ cinza de casca de arroz $(\mathrm{S}+\mathrm{C}), \mathrm{S} 2=80 \%$ solo $+20 \%$ húmus de minhoca $(\mathrm{S}+\mathrm{H}), \mathrm{S} 3=80 \%$ cinzas de casca de arroz + $20 \%$ húmus de minhoca $(\mathrm{C}+\mathrm{H}), \mathrm{S} 4=40 \%$ solo $+40 \%$ cinzas de casca de arroz $+20 \%$ húmus de minhoca $(\mathrm{S}+\mathrm{C}+\mathrm{H}), \mathrm{S} 5=100 \%$ turfa $(\mathrm{T})$, $\mathrm{S} 6=100 \%$ Mecplant $^{\mathbb{B}}(\mathrm{M}), \mathrm{S} 7=50 \%$ turfa $+50 \%$ cinzas de casca de arroz $(\mathrm{T}+\mathrm{C}) .{ }^{* *}$ Substrato $\mathrm{S}+\mathrm{C}(\mathrm{N}=44)$ e demais $(\mathrm{N}=56) .{ }^{* * *} \mathrm{Substrato}$ $\mathrm{N}+\mathrm{C}(\mathrm{N}=16)$ e demais $(\mathrm{N}=56)$.

${ }^{*} \mathrm{~S} 1=50 \%$ soil $+50 \%$ rice husk ash, $S 2=80 \%$ soil $+20 \%$ earthworm vermicompost, $S 3=80 \%$ rice husk ash $+20 \%$ earthworm vermicompost, $S 4=40 \%$ soil $+40 \%$ rice husk ash $+20 \%$ earthworm vermicompost, $S 5=100 \%$ peat, S6 $=100 \%$ commercial substrate Mecplant ${ }^{\mathbb{B}}, S 7=50 \%$ peat $+50 \%$ rice husk ash. ${ }^{* *}$ Substrate $S+C(N=44)$ and other $(N=56) .{ }^{* * *}$ Substrate $N+C(N=16)$ and other $(N=56)$. 
Do ponto de vista de técnicas experimentais, os experimentos com a cultura de cravina de jardim devem considerar o tipo de substrato a ser utilizado e as variáveis produtivas e morfológicas ao dimensionar o tamanho de amostra para uma avaliação mais precisa. Para reduzir os custos de execução de experimentos, poder-se-ia optar por tamanhos de amostras diferenciados conforme a variável a ser coletada e o substrato a ser utilizado.

\section{CONCLUSÕES}

A utilização de amostras de 44 plantas de cravina de jardim para o substrato comercial Mecplant ${ }^{\circledR}$ atenderá a precisões inferiores ou iguais a $20 \%$, para todas as variáveis avaliadas. Há variação no tamanho de amostra em relação ao substrato utilizado e a variável avaliada em plantas de cravina de jardim.

\section{REFERÊNCIAS}

BARBOSA, C.K.R.; VALADARES, S.V.; BONFIM, F.P.G.; HONORIO, I.C.G.; MARTINS, E.R. Influência do substrato e do tamanho da célula de bandejas de poliestireno expandido no desenvolvimento de mudas e produção de calêndula (Calendula officinalis L.). Revista Brasileira de Plantas Medicinais, Botucatu, v.12, n.1, p.18-22, 2010.

BELASQUE JUNIOR, J.; JACIANI, F.J.; MARIN, D.R.; BARBOSA, J.C. Tamanho da amostra para quantificação do diâmetro de lesões de cancro cítrico. Tropical Plant Pathology, Viçosa, v.33, n.04, p.317-322, 2008. DOI: $<$ http://dx.doi.org/10.1590/S1982-56762008000400010>.

BEZERRA, F.B.; OLIVEIRA, M.A.C.L.; PEREZ, D.V.; ANDRADE, A.G.; MENEGUELLI, N.A. Lodo de esgoto em revegetação de área degradada. Pesquisa Agropecuária Brasileira, Brasília, v.41, n.3, p.469-476, 2006.

BOUCHÉ, M.B. Lombriciens de France. E'cologie et Syste'matique. Annales de Zoologie Ecologie Animale. Paris: INRA, 1972. 671pp. vol 2.

CARGNELUTTI FILHO, A.; EVANGELISTA, D.H.R.; GONÇALVES, E.C.P.; STORCK, L. Tamanho de amostra de variáveis de genótipos de soja. Ciência Rural, Santa Maria, v.39, n.4, p.983-991, 2009. DOI: <http://dx.doi. org/10.1590/S0103-84782009005000016>.

CARGNELUTTI FILHO, A.; LOPES, S.J.; BRUM, B.; DA SILVEIRA, T.R.; TOEBE, M.; STORCK, L. Tamanho de amostra de variáveis em híbridos de mamoneira. Ciência Rural, Santa Maria, v.40, n.2, p.280-287, 2010a. DOI: $<$ http://dx.doi.org/10.1590/S0103-84782010000200005>.

CARGNELUTTI FILHO, A.; RIBEIRO, N.D.; STORCK, L.; JOST, E.; POERSCH, N.L. Tamanho de amostra de variáveis de cultivares de feijão. Ciência Rural, Santa Maria, v.38, n.3, p.635-642, 2008. DOI: <http://dx.doi. org/10.1590/S0103-84782008000300007>.
CARGNELUTTI FILHO, A.; TOEBE, M.; DA SILVEIRA, T.R.; CASAROTTO, G.; HAESBAERT, F.M.; LOPES, S.J. Tamanho de amostra e relações lineares de variáveis morfológicos e produtivos de crambe. Ciência Rural, Santa Maria, v.40, n.11, p.2262-2267, 2010b. DOI: <http:// dx.doi.org/10.1590/S0103-84782010001100003>.

CATAPATTI, T. R.; GONÇALVES, M.C.; NETO, M.R.S.; SOBROZA, R. Tamanho de amostra e número de repetições para avaliação de caracteres agronômicos em milho-pipoca. Ciência e Agrotecnologia, Lavras, v.32, n.3, p.855-862, 2008. DOI: <http://dx.doi.org/10.1590/ S1413-70542008000300023>.

DEY, K.P.; GHOSH, S.; NASKAR, M.K. Organic template-free synthesis of ZSM-5 zeolite particles using rice husk ash as silica source. Ceramics International, v.39, p.2153-2157, 2013.

ESTATCAMP. 2015. Portal do Action. Disponível em: $<$ http://www.portalaction.com.br $>$ Acesso em: 20 de janeiro de 2015.

FAGUNDES, J.D.; STRECK, N.A.; STORCK, L.; REINIGER, L.R.S.; KRUSE, N.D. Temperatura base, plastocrono e número final de nós no malmequer-docampo. Ciência Rural, Santa Maria, v.38, n.9, p.24712477, 2008. DOI: <http://dx.doi.org/10.1590/S0103$84782008005000021>$.

FARIA, R.T.; REGO, L.V.; BERNARDI, A.; MOLINARI, H. Performance of differents genotyps of brazilian orchid cultivation in alternatives substrates. Brazilian Archives of Biology and Technology, Curitiba, v.44, n.4, p.337342, 2001. DOI: <http://dx.doi.org/10.1590/S1516$89132001000400003>$.

FERREIRA, D.F. Estatística básica. Lavras: UFLA, 2009. $663 p$.

KÄMPF, A. Produção Comercial de Plantas Ornamentais. Porto Alegre: Agrolivros, 2005. 254p.

LOPES, J.L.W.; GUERRINI, I.A.; SAAD, J.C.C.; DA SILVA, M.R. Atributos químicos e físicos de dois substratos para produção de mudas de eucalipto. Cerne, Lavras, v. 14, n. 4, p. 358-367, 2008.

LORENTZ L. H.; LÚCIO, A.D.; BOLIGON, A.A.; LOPES, S.J.; STORCK, L. Variabilidade da produção de frutos de pimentão em estufa plástica. Ciência Rural, Santa Maria, v.35, n.2, p.316-323, 2005. DOI: <http:// dx.doi.org/10.1590/S0103-84782005000200011>.

LORENTZ, L. H.; LÚCIO, A.D.; STORCK, L.; LOPES, S.J.; BOLIGON, A.A.; CARPES, R.H. Variação temporal do tamanho de amostra para experimentos em estufa plástica. Ciência Rural, Santa Maria, v.34, n.4, p.10431049, 2004. DOI: <http://dx.doi.org/10.1590/S0103$84782004000400012>$. 
LÚCIO, A.D.; COUTO, M.R.M.; TREVISAN, J.N.; MARTINS, G.A.K.; LOPES, S.J. Excesso de zeros nas variáveis observadas: estudo de caso em experimento com brócolis. Bragantia, Campinas, v.69, n.4, p.10351046, 2010. DOI: <http://dx.doi.org/10.1590/S0006$87052010000400032>$.

LÚCIO, A.D.; SCHWERTNER, D.V.; HAESBAERT, F.M.; SANTOS, D.; BRUNES, R.R.; RIBEIRO, A.L.P.; LOPES, S.J. Violação dos pressupostos do modelo matemático e transformação de dados. Horticultura Brasileira, Brasília, v.30, p. 415-423, 2012.

MALAVOLTA, E. Manual de nutrição mineral de plantas. São Paulo: Ceres, 2006. 638p.

NUNES, G.H.S.; TORQUATO, J.E.; JÚNIOR, R.S.; FERREIRA, H.A.; NETO, F.B. Tamanho amostral para estimar o teor de sólidos solúveis totais em talhões de melão amarelo. Caatinga, Mossoró, v.19, n.2, p.117-122, 2006.

RESENDE, M.D.V. DE; SOUZA JUNIOR, C.L. DE. Número de repetições e tamanho da parcela para seleção de progênies de milho em solos sob cerrado e fértil. Pesquisa Agropecuária Brasileira, Brasília, v.32, n.8, p.781-788, 1997.

ROZANE, D.; NATALE, W.; PRADO, R. de M.; BARBOSA, J.C. Tamanho da amostra foliar para avaliação do estado nutricional de goiabeiras com e sem irrigação. Revista Brasileira de Engenharia Agrícola e Ambiental, Campina Grande, v.13, n.03, p.233-239, 2009. DOI: $<$ http://dx.doi.org/10.1590/S1415-43662009000300003>.

SAKATA SEED SUDAMERICA LTDA ${ }^{\circledR}$. 2015. Dianthus F1 Diamond. Disponível em: <http://www.sakata.com. br/index.php? action $=$ catalogo $\&$ cultura $=3 \&$ language $=p t>$ Acesso em: 25 de março de 2015.

SALVADOR, E. D.; MINAMI, K.; FARIA, M.V.; DE RESENDE, J.T.V.; MALLMANN, N. 2015. Estudo das características físicas adequadas ao crescimento de gloxínia (Sinningia speciosa). Disponível em: $<$ http://www.abhorticultura.co m.br/biblioteca/arquivos/ Download/Biblioteca/44 338.pdf>. Acesso em: 15 de outubro de 2015.
SANTOS, M.R.A.; TIMBÓ, A.L.O.; CARVALHO, A.C.P.P.; MORAIS, J.P.S. Estudo de adubos e substrato orgânicos no desenvolvimento de mudas micropropagadas de helicônia. Horticultura Brasileira, Brasília, v. 24, n. 3, p. 273-278, 2006.

SANTOS, P.M. dos.; LOPES, S.J.; LÚCIO, A.D.; SANTOS, O.S.; DOS SANTOS, V.J.; BRUM, B. Cronograma de amostragem de plantas de alface hidropônica para ajuste de curvas de crescimento. Ciência Rural, Santa Maria, v.37, n.6, p.1601-1608, 2007. DOI: <http://dx.doi.org/10.1590/ S0103-84782007000600015>.

SARANGI, M.; NAYAK, P.; TIWARI, T.N. Effect of temperature on nano-crystalline silica and carbon composites obtained from rice-husk ash. Composites Part B: Engineering, v.42, p.1994-1998, 2011.

SILVA, G.O.; VIEIRA, J.V.; VILLELA, M.S. Tamanho de amostra para avaliação de caracteres de cenoura em sistemas de cultivo agroecológico. Horticultura Brasileira, Brasília, v.27, n.2, 2009.

SOUZA, M.F.; LÚCIO, A.D.; STORCK, L.; CARPES, R.H.; SANTOS, P.M.; SIQUEIRA, L.F. Tamanho da amostra para peso da massa de frutos, na cultura da abóbora italiana em estufa plástica. Revista Brasileira de Agrociência, Pelotas, v.8, n.2, p.123-128, 2002.

STORCK, L.; FIORIN, R.A.; CARGNELUTTI FILHO, A.; GUEDES, J.V.C. A sampling procedure for quantifying mites in soybeans. Experimental and Applied Acarology, v.57,n.2,p.117-126, 2012.DOI: <http://dx.doi.org/10.1007/ S10493-012-9547-8>.

STORCK, L.; LOPES, S.J.; CARGNELUTTI FILHO, A.; MARTINI, L.F.D.; DE CARVALHO, M.P. Sample size for single, double, and three-way hybrid corn ear traits. Scientia Agricola, Piracicaba, v.64, n.1, p.30-35, 2007. DOI: $<$ http://dx.doi.org/10.1590/S0103-90162007000100005>.

VERDONCK, O.; GABRIELS, R. Substrate requirements for plants. Acta Horticulturae, Wageningen, v.221, p.1923, 1988 . 